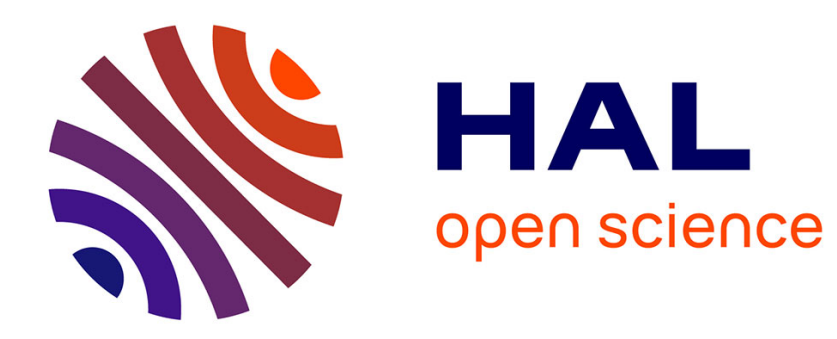

\title{
Circadian aspects of adipokine regulation in rodents
} Etienne Challet

\section{To cite this version:}

Etienne Challet. Circadian aspects of adipokine regulation in rodents. Best practice \& research. Clinical endocrinology \& metabolism, 2017, 31 (6), pp.573-582. 10.1016/j.beem.2017.09.003 . hal02557249

\section{HAL Id: hal-02557249 \\ https://hal.science/hal-02557249}

Submitted on 4 Nov 2021

HAL is a multi-disciplinary open access archive for the deposit and dissemination of scientific research documents, whether they are published or not. The documents may come from teaching and research institutions in France or abroad, or from public or private research centers.
L'archive ouverte pluridisciplinaire HAL, est destinée au dépôt et à la diffusion de documents scientifiques de niveau recherche, publiés ou non, émanant des établissements d'enseignement et de recherche français ou étrangers, des laboratoires publics ou privés. 


\section{Best Practice \& Research Clinical Endocrinology \& Metabolism}

2017 Dec;31(6):573-582.

doi: 10.1016/j.beem.2017.09.003. Epub 2017 Sep 22.

\section{Circadian aspects of adipokine regulation in rodents}

Etienne Challet, PhD, Research Director *

* Circadian Clocks and Metabolism Team, Institute of Cellular and Integrative Neurosciences, UPR3212, Centre National de La Recherche Scientifique (CNRS), University of Strasbourg, 5 rue Blaise Pascal, F-67000 Strasbourg, rance. Fax: +33 388456654 .

E-mail address: challet@inci-cnrs.unistra.fr

Most hormones display daily fluctuations of secretion during the 24-h cycle. This is also the case for adipokines, in particular the anorexigenic hormone, leptin. The temporal organization of the endocrine system is principally controlled by a network of circadian clocks. The circadian network comprises a master circadian clock, located in the suprachiasmatic nucleus of the hypothalamus,

synchronized to the ambient light, and secondary circadian clocks found in various peripheral organs, such as the adipose tissues. Besides circadian clocks, other factors such as meals and metabolic status impact daily profiles of hormonal levels. In turn, the precise daily pattern of hormonal release provides temporal signaling information. This review will describe the reciprocal links between the circadian clocks and rhythmic secretion of leptin, and discuss the metabolic impact of circadian desynchronization and altered rhythmic leptin. 


\section{Introduction}

Adipokines are cytokines produced and secreted by the adipose tissue. Leptin, adiponectin and resistin are among the best known adipokines. They all participate in the regulation of energy metabolism by acting on different tissues such as the brain, the liver, the skeletal muscle and the adipose tissue. In case of metabolic disorders, such as diet-induced obesity or insulin resistance, the production of the adipokines is modified, some of them being secreted in larger or lower quantities [1]. The 24-h organization of physiology -including the endocrine system- and behavior is controlled by a multioscillatory network comprising circadian clocks in most cells and tissues, including the adipose tissues [2]. Circadian oscillations are generated on a daily basis by a set of clock genes that work in a temporally organized manner, forming positive and negative feedback loops of transcription and translation. Briefly, the molecular clockwork relies on the clock proteins BMAL1 and CLOCK that dimerize and bind to E-box sequences in the promoters of other clock genes, including Period 1-3 and Cryptochrome 1-2 to activate their transcription. After reaching a certain concentration in the cytoplasm, PERIOD and CRYPTOCHROME proteins dimerize and translocate in the nucleus where they inhibit their own transcription mediated by CLOCK/BMAL1 dimers. Reinforcing loops comprise the circadian nuclear receptors, REV-ERB $\alpha-\beta$ and ROR $\alpha-\beta-\gamma$ that control the rhythmic transcription of Bmal1 and Clock. Post-translational modifications also contribute to the clockwork [2]. While the same clock genes are essentially used by the clock machinery in most cells, clock-controlled genes (i.e., genes whose rhythmic transcription is directly controlled by the clockwork) are specific to given tissues or even cell types. Ultimately, clock-controlled genes can also provide local temporal cues, either within or outside of the cells. The circadian network is synchronized via neuronal and hormonal signals by a master clock located in the suprachiasmatic nuclei of the hypothalamus, mainly entrained to the environment by light perceived by the retina [2,3] (Fig. 1).

This review aims at highlighting the reciprocal links between the circadian clocks and adipokines, and will be mostly focused on leptin. As will be described, the daily rhythm of leptin secretion is controlled in part by circadian clocks in the hypothalamus and adipose tissue, but also by other factors such as feeding and hormones, like glucocorticoids and melatonin. In turn, the rhythmic secretion of leptin provides time-cues within the body. Desynchronization between the master and peripheral clocks, for instance by altered timing of light exposure or meal time, favors the development of metabolic disorders [4]. Another issue that will be briefly covered is the metabolic impact of circadian desynchronization and altered rhythmic leptin. As a consequence, misalignment of adipokine signals, whatever the cause, may have a deleterious impact on circadian rhythmicity and metabolic health.

\section{Daily expression and secretion of adipokines}

The most studied adipokine is leptin, a hormone conveying information on peripheral energy status to the brain and acting on the hypothalamus to inhibit food intake. The anorectic effects of leptin are partly mediated by inhibition of neurons containing neuropeptide $Y$ and activation of proopiomelanocortin-containing neurons in the hypothalamic arcuate nuclei [5]. Leptin is also involved in peripheral metabolism of glucose and lipids. Among others, leptin promotes hepatic insulin sensitivity [6]. Leptin is rhythmically released over $24 \mathrm{~h}$. The circadian rhythm of leptin secretion in nocturnal rodents, such as rats, hamsters and mice, starts rising at night, which corresponds to the active period [7-13] (Fig.1). Synthesis of leptin in the white adipose tissue is rhythmic too, as shown by higher nocturnal levels of Leptin mRNA in various adipose depots of rats [12,14]. In diurnal rodents, leptin is mostly secreted during daytime, also corresponding to the active period $[15,16]$.

Adiponectin, another adipokine, increases insulin sensitivity and inhibits inflammatory pathways [17]. Levels of Adiponectin/AdipoQ mRNA display diurnal variations in the white adipose tissue of rats and mice with highest values in late day/early night [14,18-20]. In addition, circadian oscillations are found in cultured 3T3-L1 adipocytes [18]. Remarkably, in several rat studies, no clear daily rhythmicity of plasma adiponectinwas detected [10,20,21], although other studies did report significant day-night variations with higher diurnal values in this species [22,23]. In mice, a circadian rhythm of plasma adiponectin has been described with higher values in late night [24]. The circadian effects of adiponectin have been poorly studied. KK-T mice are genetically obese and express much less 
adiponectin. They display a shorter free-running period, which may be related to their reduced levels of adiponectin that would in turn affect the suprachiasmatic clock [25].

Resistin and visfatin are two other adipokines involved in insulin sensitivity and inflammatory processes [26]. Regarding resistin, its transcriptional expression in the white adipose tissue of rats has been found to be either rhythmic with higher values in late night [20] or not significantly rhythmic [14]. In mice, higher levels of Resistin mRNA in visceral adipose tissue are detected in late resting phase [19]. Circulating levels of resistin assayed in rats are increased during the late resting and early active periods [20]. With respect to visfatin, the gene coding this hormone is rhythmically expressed in visceral adipose tissue of mice and rats, with much higher values at night $[14,19,27]$.

\section{Control of leptin rhythm}

Adipose clock

White and brown adipose tissues display daily patterns of clock gene expression that are very close to those found in the liver [28] (Fig.1). Furthermore, pre-adipocytes and adipocytes cultured in vitro display oscillatory properties that last a few cycles, thus demonstrating that the adipose tissue contains a circadian clock that generates self-sustained oscillations. In differentiated adipocytes, expression of Leptin mRNA does not follow a circadian pattern. By contrast, leptin secretion from adipocytes cultured in vitro exhibits circadian rhythmicity [29]. Mice mutant for the two clock genes Period 1 and Period 2 present elevated concentrations of leptin over the daily cycle [30]. Another argument for a role of the adipose clock in leptin secretion comes from mice with adipocyte-specific deletion of the core clock gene Bmal1 that display increased levels of plasma leptin around midday and midnight [31]. Therefore, the daily rhythm of leptin secretion can be considered as a circadian output of the white adipose clock. The aforementioned rhythmicity of Leptin mRNA in white adipose tissue sampled ex vivo [12], which is not found in adipocytes cultured in vitro [29], suggests however that in vivo fat rhythmicity is not exclusively controlled by the local clock in white adipose tissue, but is also modulated by other upstream factors.

\section{Suprachiasmatic clock and its nervous and neuroendocrine outputs}

The involvement of the suprachiasmatic clock in the regulation of leptin rhythmicity has been initially demonstrated by lesions of the suprachiasmastic region that eliminate the daily pattern of plasma leptin levels in rodents [8,32]. In rats with suprachiasmatic lesions, leptin arrhythmicity is concomitant with constant levels at relatively high concentrations, that is, close or above peak values in intact animals [8]. The adipose clock is one of the links of the multi-oscillatory circadian network. This network is under the daily control of the suprachiasmatic clock via nervous projections, local release of diffusible molecules, and hormonal rhythms. The direct nervous projections from the suprachiasmatic nuclei are relatively short within the hypothalamus, mostly limited to the paraventricular nuclei of the hypothalamus and the subparaventricular region. The target neurons then innervate sympathetic structures in the spinal cord and pre-ganglionic parasympathetic structures in the brainstem. Therefore, the suprachiasmatic clock uses the two branches of the autonomous nervous system to distribute circadian signals to peripheral organs, such as the liver and the adrenal glands [3]. The adipose tissue follows the same neural organization (Fig. 1). While the sympathetic innervation of fat depots has been known for a long time, their parasympathetic innervation was evidenced only recently using transneuronal viral tracing [33]. Selective vagotomy of the abdominal fat pads leads to a reduction in Leptin and Resistin mRNA levels, whereas Adiponectin mRNA levels do not change. The same chirurgical treatment also increases the activity of hormonesensitive lipase, thus demonstrating the anabolic role of the vagal inputs to the white adipose tissue [33]. It should be noted that the functional role of vagal input to white adipose tissue depots has been intensively debated [34,35].

The suprachiasmatic clock also distributes temporal messages via rhythmic release of diffusible substances, such as Prokineticin 2, Transforming growth factor a and cardiotrophin-like cytokine, that have been proposed to participate in the regulation of rest/activity cycle [36], but not in hormonal rhythms [37]. Another route by which the master clock distributes circadian signals to downstream 
structures is through neuroendocrine rhythms (Fig. 1). Accordingly, circadian rhythmicity of peripheral organs can be modified by the hormonal rhythms closely controlled by the suprachiasmatic nuclei. These endocrine rhythms include adrenal glucocorticoids and pineal melatonin that act as internal Zeitgebers (i.e., internal cues that enhance synchrony within the multi-oscillatory circadian network). From a clinical point of view, because both these endocrine rhythms are tightly regulated by the suprachiasmatic nuclei and are relatively resistant to external cues, they are measured and used as robust phasemarkers of the central clock [38,39].

The daily rhythm of glucocorticoid secretion usually peaks close to the onset of the active period (i.e., at dawn and dusk in diurnal and nocturnal species, respectively). Such a timed increase in circulating glucocorticoid levelsmay help to arouse the brain for future cognitive activity and activate the peripheral organs for physical activity and foraging. More details on the functional and clinical significance of the glucocorticoid rhythm are provided in recent reviews $[38,40]$. The synchronizing effects of the glucocorticoid rhythm on adipose tissue can be experimentally assessed in vitro or in vivo. Dexamethasone, a glucocorticoid receptor agonist, applied in vitro increases Leptin mRNA in primary cultured rat adipocytes and induces phase-changes in clock gene expression of 3T3-L1 preadipocytes $[41,42]$. The daily rhythm of plasma leptin persists in adrenalectomized rats implanted with corticosterone pellets that maintain constant low levels of circulating glucocorticoids. The leptin rhythm is phase-advanced by a few hours after adrenalectomy [8]. Furthermore, adrenalectomy without corticosterone implant diminishes the amplitude of daily expression of clock genes in the epididymal white adipose tissue, Period 1 and Rev-erbo being down-regulated and Cryptochrome 2, up-regulated. It is noteworthy that mRNA levels of genes coding for Leptin, Adiponectin and Resistin are all down-regulated in the white adipose tissue of adrenalectomized rats, the expression of both Leptin and Adiponectin losing daily rhythmicity. Expression of many metabolic genes (e.g., Fatty acid synthase, Lipoprotein lipase, Hormone-sensitive lipase, Glucose transporter 4) also loses daily rhythmicity after adrenalectomy [14]. Together, these findings reveal that rhythmic glucocorticoids play a significant role in controlling the daily rhythms in metabolic and adipokine gene expression of the adipose tissue, eventually independently of the adipose clock.

Melatonin is a hormone synthesized and released at night by the pineal gland that acts as an internal time-giver [39]. In primary cultured rat adipocytes, cyclic administration of melatonin mimicking the in vivo melatonin rhythm leads to up-regulated expression of clock genes and increased secretion of leptin during the biological night [43]. The modulating effect of circulating melatonin on adipose function has been assessed in vivo in pinealectomized rodents. Pinealectomy suppresses the daily variations of most clock genes in epididymal adipose tissue, reduces leptin secretion in rats and flattens the daily rhythm of leptin in hamsters $[11,44]$. These results highlight that also the nocturnal pattern of circulating melatonin controls, in part, the daily rhythm of leptin secretion from adipose tissue.

\section{Feeding conditions}

Besides circadian factors, feeding and metabolic status have major, direct effects on leptin secretion, thereby changing the shape of the daily profile of plasma leptin (Fig. 1). Notably, food intake leads to a postprandial secretion of leptin $[7,45]$. Conversely, the nocturnal peak of leptin secretion in rats and mice is completely abolished by fasting $[9,45]$. Lactation in rodents is a situation of high-energy demand coupled with hyperphagia that is also associated with a blunted nocturnal peak of plasma leptin [46]. Rodents fed with high-fat diet ad libitum display increased leptinemia, together with a disappearance of daily rhythmicity $[22,23,47]$.

Meal time exerts powerful synchronizing effects on timing of peripheral tissues, as shown by phaseshifts in peripheral clocks when food access is repeatedly limited to unusual times (i.e., resting phase) $[48,49]$. Accordingly, white and brown adipose clocks are shifted by temporal restricted feeding, as shown by phase-inversion of daily patterns of clock gene expression in nocturnal rats fed only during daytime [28]. The same protocol also inverts the daily rhythm of leptin, which then peaks during daytime in both rats and mice $[7,45,50,51]$. When food-restricted animals are exposed to a light-dark cycle, the peripheral clocks then become synchronized to feeding time and uncoupled from the suprachiasmatic nuclei that remain synchronized to light $[48,49]$. 
To remove the synchronizing effect of the feeding/fasting cycle, animals can be exposed to six-mealsper-day feeding schedules ( 1 short access to food every $4 \mathrm{~h}$ ) that prevent the daily periodicity of feeding. In rats fed with six meals per day, levels of Leptin mRNA in epididymal adipose tissue are rhythmically expressed during the light-dark cycle [14]. Furthermore, under six-meals-per-day schedule, a daily rhythm of plasma leptin is maintained, albeit phase-advanced as compared to that of control animals fed ad libitum [8]. The combination of six-meals-per-day feeding schedule and adrenalectomy leads to arrhythmicity of all clock genes and most metabolic and adipokine genes in white adipose tissue [27]. To sum up, overt rhythmicity of adipokine synthesis relies on complex interactions between the adipose clock, the suprachiasmatic clock sending timing signals via nervous and hormonal routes, and feeding/fasting cues.

\section{Timing role of leptin on circadian rhythms}

As mentioned above, at least two hormonal rhythms (i.e., glucocorticoids and melatonin) participate in the circadian organization of the body. Several lines of evidence indicate that the daily secretion of leptin on its turn also has time-giving properties on physiology and behavior. Genetic models with impaired leptin signaling have been instrumental to support this notion. They include mice lacking leptin (i.e., ob/ob mice) and rodents with mutated leptin receptor (i.e., $d b / d b$ mice and $f a / f a$ Zucker rats). All these models develop morbid obesity and more or less severe type 2 diabetes.

\section{Peripheral clocks}

$\mathrm{Ob} / \mathrm{ob}$ mice display impairments in liver and adipose clocks, characterized by dampened oscillations of clock and clock-controlled genes. Importantly, these alterations occur prior to metabolic disorders, thus raising the possibility that circadian perturbations could be due to the absence of leptin. In support of this hypothesis, treatment with exogenous leptin partially rescues the amplitude of clock gene oscillations in the peripheral tissues of $o b / o b$ mice [52]. In $f a / f a$ rats, alterations in rhythmic expression of clock genes are also found in the liver, but not in other tissues, such as mesenteric adipose tissue and heart [53] (Fig. 2).

\section{Master clock}

Leptin has been shown to modulate the electrical activity of the suprachiasmatic nuclei maintained in vitro. First, the daily firing rate of hypothalamic slices containing the master clock is dose-dependently phase-advanced by leptin during most of the circadian cycle [54]. Second, spike coding of suprachiasmatic neurons is also modified by leptin application [55], therefore suggesting that leptin conveys metabolic signals to the master clock. In vivo systemic or central injections of leptin, however, do not produce phase-shifts of the rest-activity rhythm controlled by the master clock $[45,56]$. In contrast, leptin treatment does modulate light synchronization of the suprachiasmatic clock (Fig. 2). More specifically, leptin potentiates light-induced phase-advances in wild-type mice and normalizes the increase of light-induced phase-delays in ob/ob mice [56-58]. Mapping leptin-induced P-STAT3 in the hypothalamus suggests that in vivo leptin modulates indirectly the activity of the suprachiasmatic nuclei, likely via the mediobasal hypothalamus [57].

\section{Feeding and related behavioral rhythms}

Leptin is well-known to modulate the timing of food intake by its anorectic effects mostly mediated by the arcuate nuclei of the hypothalamus. Accordingly, damage to the arcuate neurons expressing leptin receptors by local injections of saporin conjugated to leptin leads to hyperphagia coupled to arrhythmic pattern of feeding [59]. On a short-term scale, leptin modulates the temporal structure of feeding as shown, for instance, with intracerebroventricular injections of leptin in Wistar rats that reduce meal frequency and prolong the inter-meal interval, without affecting meal size [60]. On a circadian time-scale, both $d b / d b$ mice and $f a / f a$ rats display increased feeding during the resting period (daytime) that explains most of their hyperphagic phenotype [13,61]. By contrast, hyperphagia in $o b / o b$ mice takes place essentially during the active nocturnal period, suggesting a permissive role of leptin on the phase of feeding rhythm. It is noteworthy, however, that repeated injections of leptin in 
$o b / o b$ mice are able to induce time-dependent phase-shifts in the daily rhythm of food intake, thus highlighting a chronomodulatory role of leptin for that rhythmic behavior [13] (Fig. 2).

When food access is limited to a few hours during the usual resting period (i.e., daytime for nocturnal rodents) during several consecutive days, a bout of locomotor activity, so-called foodanticipatory activity, occurs before the expected meal time. Such behavioral activation prior to food access is thought to be controlled by a network of circadian clocks entrained by meal time [62]. As already mentioned, daytime restricted feeding triggers a diurnal peak of plasma leptin that starts to rise during food access $[7,45,51]$. The leptin cues modulate food anticipation because rodents with impaired leptin signaling (i.e., $f a / f a$ rats and $o b / o b$ mice) display increased food-anticipatory activity, while leptin treatment in food-restricted ob/ob mice prevents its expression [63,64] (Fig. 2).

\section{Glucose rhythm}

Leptin may also participate in the control of daily rhythm of glucose (Fig. 2), as demonstrated by inverted daily rhythm of plasma glucose in mice with impaired leptin signaling (i.e., $o b / o b$ and $d b / d b$ mice) as compared to respective control littermates. In addition, repeated treatments with leptin during nighttime lead to phase-advanced rhythm of plasma glucose, while daytime leptin injections cause arrhythmicity of plasma glucose in $o b / o b$ mice. Of note, the daily variations of circulating lipids (e.g., non-esterified fatty acids and high-density lipoproteins) remain unchanged in both $o b / o b$ and $d b / d b$ mice [13]. The location (central and/or peripheral) of the targets and the mechanisms by which leptin controls glucose production and/or uptake to change glucose rhythm remain to be identified. Nevertheless, the differential changes in the daily timing of glucose and lipid metabolism indicate that impaired leptin signaling is associated with an internal desynchronization between daily rhythms of plasma glucose and lipids.

\section{Circadian desynchronization and leptin}

Circadian alterations can be caused either by an altered endogenous clockwork and/or an altered rhythmic environment. Multiple interactions have been demonstrated between clock genes/proteins and essential factors of the intracellular metabolism, such as SIRT1 and AMPK $[65,66]$. As a consequence, genetic deletion of clock genes leads in most cases to metabolic disturbances, which in turn impinge directly or indirectly on leptin regulation. For instance, mice carrying a dominant negative mutation of the circadian gene Clock are hyperphagic, hypoactive, overweight, and display increased levels of plasma leptin during daytime under chow diet and more severe hyperleptinemia under highfat diet, as compared to respective wild-type control animals [67]. Mice bearing a Period 1/Period 2 double mutation also show constitutive daily hyperleptinemia [30]. Moreover, mice lacking the RevErb $\alpha$ gene become obese and hyperleptinemic under both chow and high-fat diet, even if they eat the same quantity of energy as wild-type mice [68]. Mice lacking Bmal1 specifically in adipocytes are also overweight and show higher leptinemia around midday and midnight [31].

Circadian clocks being synchronized to ambient light and feeding time, alterations in these rhythmic cues, such as chronic changes in timing of lightedark cycles (chronic jetlag) or behavioral activity and meals occurring during the usual resting period (shift-work), can induce desynchronization. A number of epidemiological studies have found positive correlations between circadian desynchronization induced by shift-work and increased risks to develop the metabolic syndrome $[4,69,70]$. In animal studies also, chronic states of circadian desynchronization (e.g., due to impaired synchronization of the master clock to the lighting conditions, mistimed meals or impaired coupling between the master clock and peripheral oscillators) have deleterious impact on metabolic health. In rats, chronic jet-lag by weekly light-dark shifts increases energy intake and reduce locomotor activity, leading to higher body mass gain [71] and impaired insulin regulation [72]. Diurnal rodents exposed to similar chronic jet-lag do not become overweight, but develop glucose intolerance [73]. Nocturnal rats forced to be aroused during daytime show arrhythmicity in plasma glucose and inverted rhythm of triglycerides [74]. Furthermore, chronic sleep debt has been recognized as a risk factor for obesity and diabetes [75]. Mice sleep-deprived during their early resting period are hyperphagic and have higher secretion of leptin at night [76]. 
The functional significance of rhythmic leptin has been investigated in $o b / o b$ mice challenged with a specific condition of circadian desynchronization, namely, mistimed food access (daytime). Ob/ob mice receiving a non-rhythmic constant release of leptin gain similar body mass, independently of whether they are fed during daytime or nighttime. This indicates that leptin alone does not modulate mass gain. By contrast, $o b / o b$ mice receiving a rhythmic leptin treatment gain more body mass when they are fed during daytime, compared to the animals fed at night [50]. This study points out that mistimed feeding interacts with the daily rhythm of leptin to influence adiposity.

Altogether, these findings reveal that circadian desynchronization, in close relation with leptin rhythm, is an aggravating factor for the development of obesity and/or diabetes.

\section{Summary}

Leptin release from the adipose tissue is rhythmic on a daily basis. On the one hand, the leptin rhythm is regulated by the adipose clockwork and by timing cues coming from the master suprachiasmatic clock through autonomous nervous signals and neuroendocrine rhythms (i.e., adrenal glucocorticids and pineal melatonin), while feeding and metabolic status also markedly affect the shape of rhythmic leptin. On the other hand, plasma leptin cues distribute temporal messages to various physiological and behavioral outcomes, including clock function, glucose rhythm, feeding/fasting cycle and food anticipation. Circadian desynchronization has pathophysiological consequences on metabolic health, and altered rhythmic leptin may play a role in that respect. A better insight into the chronomodulatory of leptin on circadian clocks and glucose metabolism will be relevant for developing chronotherapeutic endocrine strategies.

\section{Practice points}

- The adipose tissues contain local circadian clocks.

- Leptin is rhythmically expressed and released during the 24-h cycle.

- Leptin rhythm is controlled by a combination of cues coming from the adipose clock, the master hypothalamic clock, and feeding pattern.

- Rhythmic leptin participates in the circadian organization of the body.

\section{Research agenda}

- Determination of the mechanisms by which the clockwork controls transcriptional rhythmicity of adipokines.

- Characterization of the chronomodulatory role of leptin on glucose production versus uptake.

- To assess in leptin-deficient mice the biological impact of restoring a physiological rhythm of leptin, rather than using timed injections.

- Development of chronopharmacological strategies targeting leptin receptors to treat metabolic disorders associated with circadian desynchronization.

\section{References}

[1] Ahima RS, Lazar MA. Adipokines and the peripheral and neural control of energy balance. Mol Endocrinol 2008;22: $1023 e 31$.

[2] Mohawk JA, Green CB, Takahashi JS. Central and peripheral circadian clocks in mammals. Annu Rev Neurosci 2012;35: $445 \mathrm{e} 62$.

[3] Kalsbeek A, Palm IF, La Fleur SE, et al. SCN outputs and the hypothalamic balance of life. J Biol Rhythms 2006;21:458e69. *[4] Arble DM, Bass J, Behn CD, et al. Impact of sleep and circadian disruption on energy balance and diabetes: a summary of workshop discussions. Sleep 2015;38:1849e60.

[5] Morton GJ, Schwartz MW. Leptin and the central nervous system control of glucose metabolism. Physiol Rev 2011;91: $389 \mathrm{e} 411$.

[6] Lam NT, Cheung AT, Riedel MJ, et al. Leptin reduces glucose transport and cellular ATP levels in INS-1 beta-cells. J Mol Endocrinol 2004;32:415e24.

[7] Ahima RS, Prabakaran D, Flier JS. Postnatal leptin surge and regulation of circadian rhythm of leptin by feeding. Implications for energy homeostasis and neuroendocrine function. J Clin Invest 1998;101:1020e7.

*[8] Kalsbeek A, Fliers E, Romijn JA, et al. The suprachiasmatic nucleus generates the diurnal changes in plasma leptin levels. Endocrinology 2001;142:2677e85. 
[9] Ahren B. Diurnal variation in circulating leptin is dependent on gender, food intake and circulating insulin in mice. Acta Physiol Scand 2000;169:325e31.

[10] Bertani S, Carboni L, Criado A, et al. Circadian profile of peripheral hormone levels in SpragueeDawley rats and in common marmosets (Callithrix jacchus). In Vivo 2010;24:827e36.

[11] Chakir I, Dumont S, Pevet P, et al. Pineal melatonin is a circadian time-giver for leptin rhythm in Syrian hamsters. Front Neurosci 2015;9:190.

[12] Sanchez J, Oliver P, Pico C, et al. Diurnal rhythms of leptin and ghrelin in the systemic circulation and in the gastric mucosa are related to food intake in rats. Pflugers Arch 2004;448:500e6.

*[13] Grosbellet E, Dumont S, Schuster-Klein C, et al. Leptin modulates the daily rhythmicity of blood glucose. Chronobiol Int 2015;32:637e49.

[14] Su Y, van der Spek R, Foppen E, et al. Effects of adrenalectomy on daily gene expression rhythms in the rat suprachiasmatic and paraventricular hypothalamic nuclei and in white adipose tissue. Chronobiol Int 2015;32:211e24.

[15] Cuesta M, Clesse D, Pevet P, et al. From daily behavior to hormonal and neurotransmitters rhythms: comparison between diurnal and nocturnal rat species. Horm Behav 2009;55:338e47.

[16] Chakir I, Dumont S, Pevet P, et al. The circadian gene Clock oscillates in the suprachiasmatic nuclei of the diurnal rodent Barbary striped grass mouse, Lemniscomys barbarus: a general feature of diurnality? Brain Res 2015;1594:165e72.

[17] Stefan N, Stumvoll M. Adiponectin e its role in metabolism and beyond. Horm Metab Res 2002;34:469e74.

[18] Barnea M, Chapnik N, Genzer Y, et al. The circadian clock machinery controls adiponectin expression. Mol Cell Endocrinol 2015;399:284e7.

[19] Ando H, Yanagihara H, Hayashi $Y$, et al. Rhythmic messenger ribonucleic acid expression of clock genes and adipocytokines in mouse visceral adipose tissue. Endocrinology 2005;146:5631e6.

[20] Oliver P, Ribot J, Rodriguez AM, et al. Resistin as a putative modulator of insulin action in the daily feeding/fasting rhythm. Pflugers Arch 2006;452:260e7.

[21] Sukumaran S, Jusko WJ, DuBois DC, et al. Mechanistic modeling of the effects of glucocorticoids and circadian rhythms on adipokine expression. J Pharmacol Exp Ther 2011;337:734e46.

[22] de Oliveira C, Scarabelot VL, de Souza A, et al. Obesity and chronic stress are able to desynchronize the temporal pattern of serum levels of leptin and triglycerides. Peptides 2014;51:46e53.

[23] Cano P, Cardinali DP, Rios-Lugo MJ, et al. Effect of a high-fat diet on 24-hour pattern of circulating adipocytokines in rats. Obesity (Silver Spring) 2009;17:1866e71.

[24] Rudic RD, McNamara P, Curtis AM, et al. BMAL1 and CLOCK, two essential components of the circadian clock, are involved in glucose homeostasis. PLoS Biol 2004;2:e377.

[25] Hashinaga T, Wada N, Otabe S, et al. Modulation by adiponectin of circadian clock rhythmicity in model mice for metabolic syndrome. Endocr J 2013;60:483e92.

[26] Stofkova A. Resistin and visfatin: regulators of insulin sensitivity, inflammation and immunity. Endocr Regul 2010;44: $25 e 36$.

[27] Su Y, Foppen E, Zhang Z, et al. Effects of 6-meals-a-day feeding and 6-meals-a-day feeding combined with adrenalectomy on daily gene expression rhythms in rat epididymal white adipose tissue. Genes Cells 2016;21:6e24.

*[28] Zvonic S, Ptitsyn AA, Conrad SA, et al. Characterization of peripheral circadian clocks in adipose tissues. Diabetes 2006; 55:962e70.

*[29] Otway DT, Frost G, Johnston JD. Circadian rhythmicity in murine pre-adipocyte and adipocyte cells. Chronobiol Int 2009; 26:1340e54.

[30] Fu L, Patel MS, Bradley A, et al. The molecular clock mediates leptin-regulated bone formation. Cell 2005;122:803e15. [31] Paschos GK, Ibrahim S, Song WL, et al. Obesity in mice with adipocyte-specific deletion of clock component Arntl. Nat Med 2012;18:1768e77.

[32] Karakas A, Gunduz B. Suprachiasmatic nuclei may regulate the rhythm of leptin hormone release in Syrian hamsters (Mesocricetus auratus). Chronobiol Int 2006;23:225e36.

*[33] Kreier F, Fliers E, Voshol PJ, et al. Selective parasympathetic innervation of subcutaneous and intra-abdominal fatefunctional implications. J Clin Invest 2002;110:1243e50.

[34] Giordano A, Song CK, Bowers RR, et al. White adipose tissue lacks significant vagal innervation and immunohistochemical evidence of parasympathetic innervation. Am J Physiol Regul Integr Comp Physiol 2006;291:R1243e55.

[35] Kreier F, Buijs RM. Evidence for parasympathetic innervation of white adipose tissue, clearing up some vagaries. Am J Physiol Regul Integr Comp Physiol 2007;293:R548e9 [Author reply R50e2, discussion R53eR54].

[36] Li JD, Hu WP, Zhou QY. The circadian output signals from the suprachiasmatic nuclei. Prog Brain Res 2012;199:119e27.

[37] Meyer-Bernstein EL, Jetton AE, Matsumoto SI, et al. Effects of suprachiasmatic transplants on circadian rhythms of neuroendocrine function in golden hamsters. Endocrinology 1999;140:207e18.

[38] Oster H, Challet E, Ott V, et al. The functional and clinical significance of the 24-hour rhythm of circulating glucocorticoids. Endocr Rev 2017;38:3e45.

[39] Pevet P, Challet E. Melatonin: both master clock output and internal time-giver in the circadian clocks network. J Physiol Paris 2011;105:170e82.

[40] Kalsbeek A, van der Spek R, Lei J, et al. Circadian rhythms in the hypothalamo-pituitary-adrenal (HPA) axis. Mol Cell Endocrinol 2012;349:20e9.

*[41] Alonso-Vale MI, Andreotti S, Borges-Silva C, et al. Intermittent and rhythmic exposure to melatonin in primary cultured adipocytes enhances the insulin and dexamethasone effects on leptin expression. J Pineal Res 2006;41:28e34. 
[42] Barnea M, Sherman H, Genzer Y, et al. Association between phase shifts, expression levels, and amplitudes in peripheral circadian clocks. Chronobiol Int 2013;30:618e27.

[43] Alonso-Vale MI, Andreotti S, Mukai PY, et al. Melatonin and the circadian entrainment of metabolic and hormonal activities in primary isolated adipocytes. J Pineal Res 2008;45:422e9.

[44] de Farias Tda S, de Oliveira AC, Andreotti S, et al. Pinealectomy interferes with the circadian clock genes expression in white adipose tissue. J Pineal Res 2015;58:251e61.

[45] Martinez-Merlos MT, Angeles-Castellanos M, Diaz-Munoz M, et al. Dissociation between adipose tissue signals, behavior and the food-entrained oscillator. J Endocrinol 2004;181:53e63.

[46] Denis RG, Williams G, Vernon RG. Regulation of serum leptin and its role in the hyperphagia of lactation in the rat. J Endocrinol 2003;176:193e203.

[47] Kohsaka A, Laposky AD, Ramsey KM, et al. High-fat diet disrupts behavioral and molecular circadian rhythms in mice. Cell Metab 2007;6:414e21.

[48] Damiola F, Le Minh N, Preitner N, et al. Restricted feeding uncouples circadian oscillators in peripheral tissues from the central pacemaker in the suprachiasmatic nucleus. Genes Dev 2000;14:2950e61.

[49] Stokkan KA, Yamazaki S, Tei H, et al. Entrainment of the circadian clock in the liver by feeding. Science 2001;291:490e3.

*[50] Arble DM, Vitaterna MH, Turek FW. Rhythmic leptin is required for weight gain from circadian desynchronized feeding in the mouse. PLoS One 2011;6:e25079.

[51] Bodosi B, Gardi J, Hajdu I, et al. Rhythms of ghrelin, leptin, and sleep in rats: effects of the normal diurnal cycle, restricted feeding, and sleep deprivation. Am J Physiol Regul Integr Comp Physiol 2004;287:R1071e9.

*[52] Ando H, Kumazaki M, Motosugi Y, et al. Impairment of peripheral circadian clocks precedes metabolic abnormalities in ob/ob mice. Endocrinology 2011;152:1347e54.

[53] Motosugi Y, Ando H, Ushijima K, et al. Tissue-dependent alterations of the clock gene expression rhythms in leptinresistant Zucker diabetic fatty rats. Chronobiol Int 2011;28:968e72.

[54] Prosser RA, Bergeron HE. Leptin phase-advances the rat suprachiasmatic circadian clock in vitro. Neurosci Lett 2003;336: $139 \mathrm{e} 42$.

[55] Inyushkin AN, Bhumbra GS, Dyball RE. Leptin modulates spike coding in the rat suprachiasmatic nucleus. J Neuroendocrinol 2009;21:705e14.

[56] Mendoza J, Lopez-Lopez C, Revel FG, et al. Dimorphic effects of leptin on the circadian and hypocretinergic systems of mice. J Neuroendocrinol 2011;23:28e38.

[57] Grosbellet E, Gourmelen S, Pevet P, et al. Leptin normalizes photic synchronization in male ob/ob mice, via indirect effects on the suprachiasmatic nucleus. Endocrinology 2015;156:1080e90.

[58] Sans-Fuentes MA, Diez-Noguera A, Cambras T. Light responses of the circadian system in leptin deficient mice. Physiol Behav 2010;99:487e94.

[59] Li AJ, Wiater MF, Oostrom MT, et al. Leptin-sensitive neurons in the arcuate nuclei contribute to endogenous feeding rhythms. Am J Physiol Regul Integr Comp Physiol 2012;302:R1313e26.

*[60] Zorrilla EP, Inoue K, Valdez GR, et al. Leptin and post-prandial satiety: acute central leptin more potently reduces meal frequency than meal size in the rat. Psychopharmacology (Berl) 2005;177:324e35.

[61] Mistlberger RE, Lukman H, Nadeau BG. Circadian rhythms in the Zucker obese rat: assessment and intervention. Appetite 1998;30:255e67.

[62] Mistlberger RE. Neurobiology of food anticipatory circadian rhythms. Physiol Behav 2011;104:535e45.

[63] Mistlberger RE, Marchant EG. Enhanced food-anticipatory circadian rhythms in the genetically obese Zucker rat. Physiol Behav 1999;66:329e35.

[64] Ribeiro AC, Ceccarini G, Dupre C, et al. Contrasting effects of leptin on food anticipatory and total locomotor activity. PLoS One 2011;6:e23364.

[65] Masri S, Orozco-Solis R, Aguilar-Arnal L, et al. Coupling circadian rhythms of metabolism and chromatin remodelling. Diabetes Obes Metab 2015;17(Suppl. 1):17e22.

[66] Jordan SD, Lamia KA. AMPK at the crossroads of circadian clocks and metabolism. Mol Cell Endocrinol 2013;366:163e9.

[67] Turek FW, Joshu C, Kohsaka A, et al. Obesity and metabolic syndrome in circadian Clock mutant mice. Science 2005;308: $1043 \mathrm{e} 5$.

[68] Delezie J, Dumont S, Dardente H, et al. The nuclear receptor REV-ERBalpha is required for the daily balance of carbohydrate and lipid metabolism. FASEB J 2012;26:3321e35.

[69] Reutrakul S, Knutson KL. Consequences of circadian disruption on cardiometabolic health. Sleep Med Clin 2015;10: $455 \mathrm{e} 68$.

[70] Evans JA, Davidson AJ. Health consequences of circadian disruption in humans and animal models. Prog Mol Biol Transl Sci 2013;119:283e323.

[71] Tsai LL, Tsai YC, Hwang K, et al. Repeated light-dark shifts speed up body weight gain in male F344 rats. Am J Physiol Endocrinol Metab 2005;289:E212e7.

[72] Bartol-Munier I, Gourmelen S, Pevet P, et al. Combined effects of high-fat feeding and circadian desynchronization. Int J Obes (Lond) 2006;30:60e7.

[73] Grosbellet E, Zahn S, Arrive M, et al. Circadian desynchronization triggers premature cellular aging in a diurnal rodent. FASEB J 2015;29:4794e803.

[74] Salgado-Delgado R, Angeles-Castellanos M, Buijs MR, et al. Internal desynchronization in a model of night-work by forced activity in rats. Neuroscience 2008;154:922e31. 
[75] Spiegel K, Tasali E, Leproult R, et al. Effects of poor and short sleep on glucose metabolism and obesity risk. Nat Rev Endocrinol 2009;5:253e61.

[76] Husse J, Hintze SC, Eichele G, et al. Circadian clock genes Per1 and Per2 regulate the response of metabolism-associated transcripts to sleep disruption. PLoS One 2012;7:e52983. 


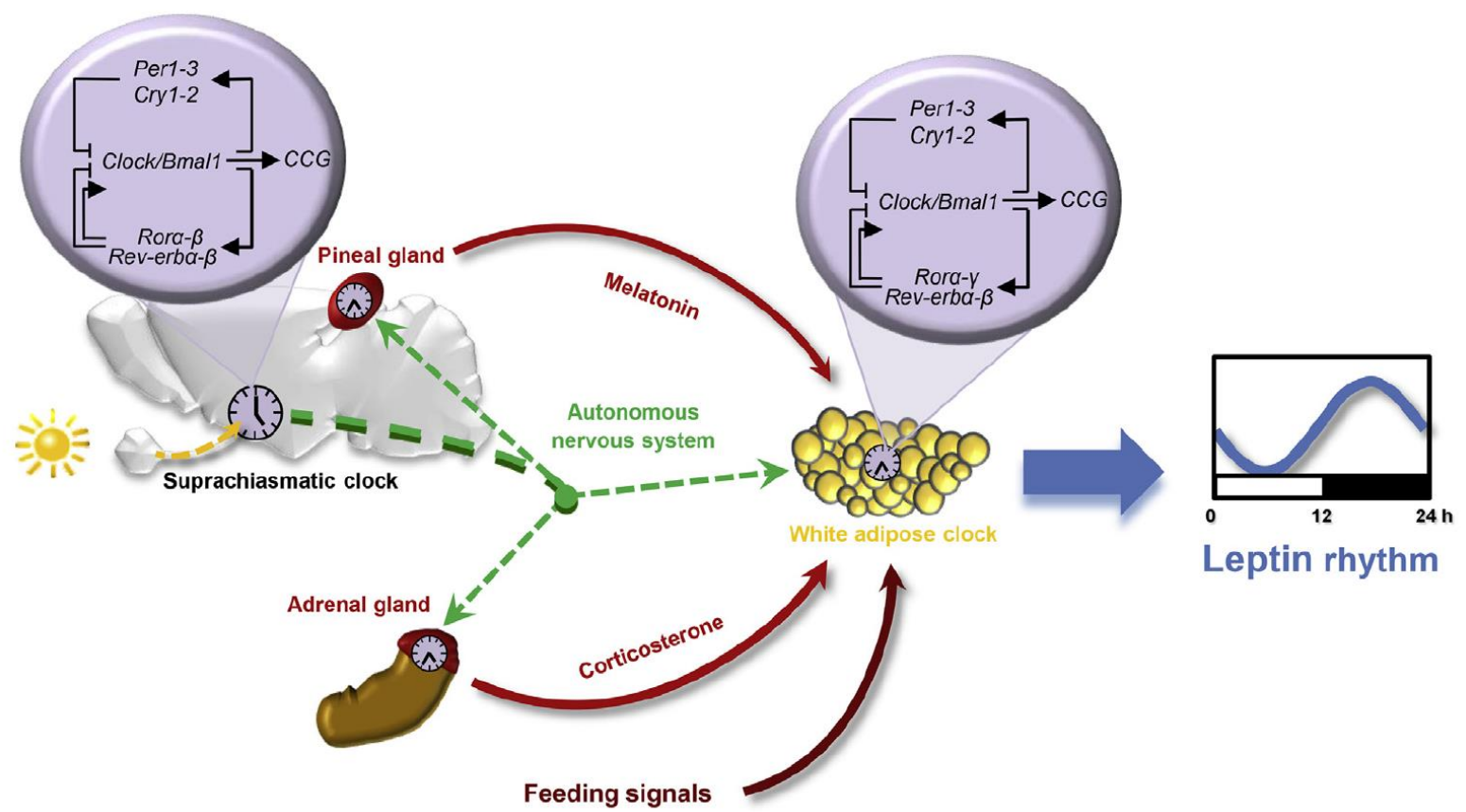

Fig. 1. Schematic overview of the control of the circadian rhythm of plasma leptin in nocturnal rodents. The circadian rhythm of leptin secretion is controlled by the local circadian clock in the adipocytes, by temporal cues from the master suprachiasmatic clock via the autonomous nervous system, hormonal rhythms (i.e., pineal melatonin and adrenal glucocorticoids such as corticosterone), and by feeding signals. The insets illustrate a simplified model of the molecular clockwork. Per, Period; Cry, Cryptochromes; CCG, clock-controlled genes. 


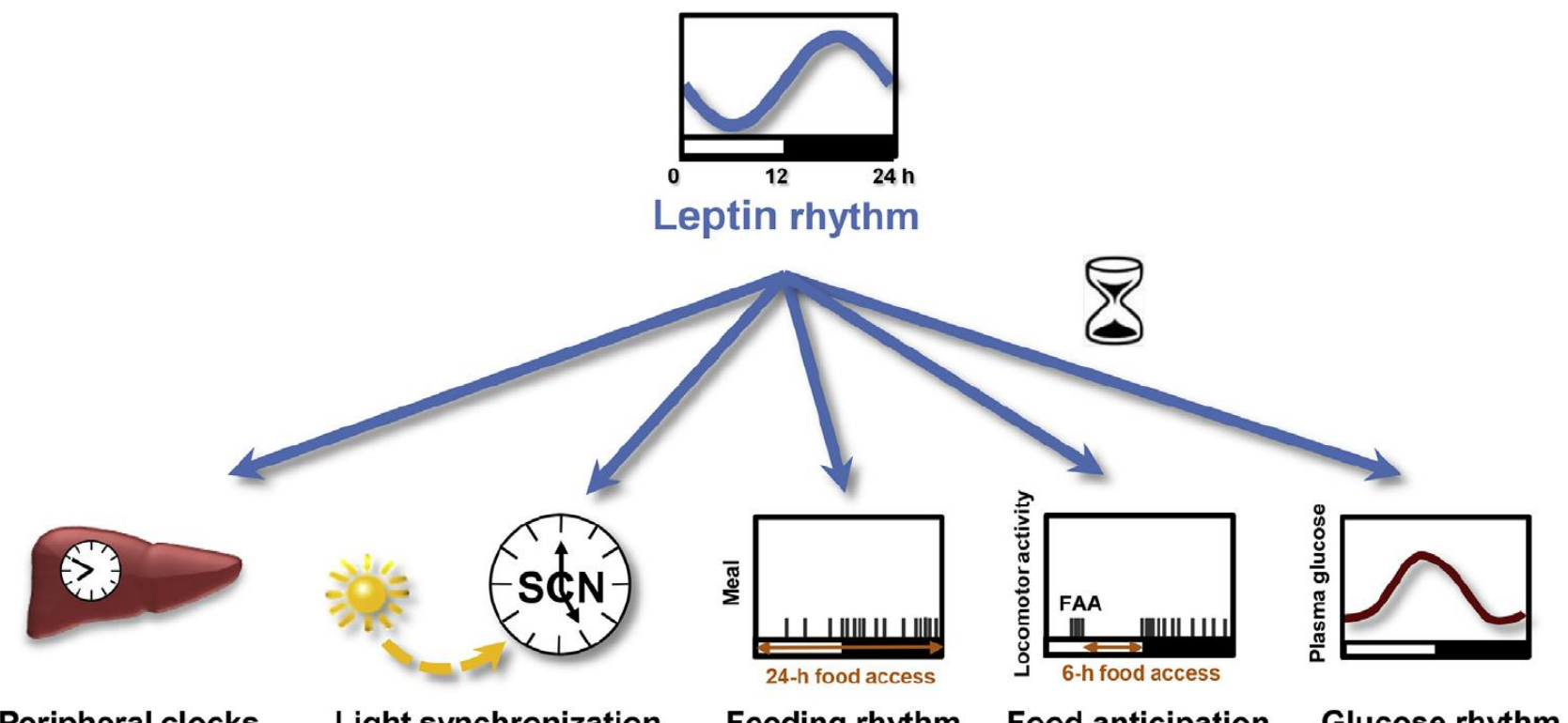

Peripheral clocks Light synchronization Feeding rhythm Food anticipation Glucose rhythm

Fig. 2. Timing role of leptin on circadian rhythms in rodents. Rhythmic leptin participates in the circadian organization of physiology and behavior by modulating (1) the peripheral clockwork, (2) light synchronization of the master clock in the suprachiasmatic nuclei (SCN), (3) the temporal structure of feeding in rodents fed ad libitum, (4) the expression of food-anticipatory activity (FAA) in rodents exposed to temporal restricted feeding, and (5) the daily profile of plasma glucose. 